CASE REPORT

\title{
Covid-19 Reinfection Dynamics-Is Immunity Short Lasting? Case Series of Healthcare Workers
}

\author{
Alamgir Khan, Manohar Kachare, Nagesh Mane, Avinash Ade \\ Department of Radiodiagnosis, Government Medical College Miraj, Pandharpur Road, Maji Sainik Vasahat, Miraj, \\ Maharashtra, India
}

\begin{abstract}
The rapidly spreading Covid-19 pandemic resulted in more than 4.7 million cases diagnosed and 78,727 deaths on September 14, 2020. SARS-CoV-2 was first expected to induce a monophasic disease with at least transient immunity. Here we report a case series of five cases of RT-PCR proved reinfection of Covid-19 in health care workers. On returning to work they might experience a Covid-19 "recurrence" or "reactivation" with increased severity of symptoms after a median symptom-free interval requiring hospitalization which raises the question is the immunity short-lasting. Further studies are required to fully understand the effect of Covid-19 on the immunity of humans in the community. J Microbiol Infect Dis 2021; 11(2):105-107.
\end{abstract}

Keywords: Covid-19, Severe acute respiratory syndrome coronavirus 2, Pandemic, Recurrence, Coronavirus infection

\section{INTRODUCTION}

This study aimed at summarizing clinical and virologic data of the case series of the patients presenting a second confirmed Covid-19 episode, at least 4 weeks after the first onset, and after a symptom-free interval at our institute.

A Covid-19 episode was defined by (i) at least one recent major clinical sign of Covid-19 including fever or chills, febrile flu-like syndrome, dyspnea, anosmia, or dysgeusia; and (ii) a positive SARS-CoV-2 RT-PCR test.

Between 23 July 2020 to 26 September 2020, 5 patients were identified all being health care workers. The median duration of symptoms was 16 days for the first episode and 12 days for the second episode. 3 patients eventually recovered and 2 patients are still under treatment.

\section{CASES}

We report a case series of five cases of RT-PCR has proven reinfection of Covid-19 in health care workers of which three were women and two were men. The median duration of symptoms was 16 (range 13-18) days for the first episode and 10 (range 5-13) days for the second one for the 3 patients who eventually recovered and two patients are still under treatment. Two patients one male and female were asymptomatic but had a positive history of contact with RT-PCR proven Covid-19 patient. The remaining three patients had flu-like symptoms for a mean duration of 4 days before being RT-PCR Covid19 swab positive. All five cases reported in our institute are health care workers. The RT PCR proven reinfection of the series of cases was approximately 4 weeks apart.

Three healthcare workers (Cases 2, 3 and 5) without significant comorbidity had a first mild Covid-19 episode with a complete recovery: On returning to work in Covid units, they might have possible Covid-19 re-exposure at work they experienced a clinical relapse requiring hospitalization with increased in severity of symptoms after a median symptom-free interval of 4 weeks and where treated with Favipiravir at both the episodes with two (Cases 2 and 3) being recovered symptomatically. The patient (case 5) still being under treatment as he still being symptomatic.

\footnotetext{
Correspondence: Dr. Alamgir Khan, Department of Radiodiagnosis, Government Medical College Miraj, Pandharpur Road, Maji Sainik Vasahat, Miraj, Maharashtra, India Email: khanmoin13077@gmail.com Received: 06 October 2020 Accepted: 05 May 2021 
The remaining two health care workers (Case 1 and 4) being asymptomatic during the first episode of Covid-19 underwent home quarantine but on returning to work in Covid units. They also might have possible Covid-19 re-exposure at work both the patients had clinical symptoms requiring hospitalization and were treated with Remdesivir (Case 1) and Favipiravir (Case 4).

\section{The clinical and the treatment data:}

Case 1 had comorbidity of Diabetes mellitus type II and Chronic Kidney Disease that required ICU hospitalization for the $2^{\text {nd }}$ episode, with the patient still being under treatment. He required oxygen therapy during the second episode.

Case 2 had a fever, myalgia, and diarrhea for 4 days in the 1st episode and was treated with Favipiravir and was declared clinically cure on Day 13 from the onset of symptoms in the 1st episode. In the 2nd episode i.e., Day 36 from day 1 of onset of symptoms from 1st episode the same patient had myalgia, fever, anosmia, and diarrhea for 5 days and again treated with Favipiravir and had duration of a total of 49 days from Day 1 of onset of symptoms in 1st episode.

Case 3 had Cough, cold, sore throat, and fever for 2 days in the 1st episode and was treated with Favipiravir and was declared clinically cure on Day 18 from the onset of symptoms in the 1st episode. In the 2nd episode i.e., Day 39 from day 1 of onset of symptoms from 1st episode the patient had Cough and fever for 2 days and again treated with Tab. Favipiravir and had a duration of a total of 44 days from Day 1 of onset of symptoms in 1st episode.

Case 4 was asymptomatic and underwent home quarantine and was declared clinically cure on Day 16 in the 1st episode. In the 2nd episode i.e., Day 35 from day 1 of from 1 st episode the patient had cough, cold, myalgia, and fever for 4 days and was treated with Tab. Favipiravir and had a duration of a total of 48 days from Day 1 of $1^{\text {st }}$ episode.

Case 5 had fever and cough for 2 days in the 1st episode and was treated with Favipiravir and was declared clinically cure on Day 16 from the onset of symptoms in the 1st episode. In the 2 nd episode i.e., Day 43 from day 1 of onset of symptoms from the 1st episode the patient had cough, cold, sore throat, and fever for 3 days and again treated with Tab. Favipiravir and is ongoing undertreatment and had a duration of a total of 44 days from Day 1 of onset of symptoms in 1st episode.

\section{The laboratory findings:}

In case 1 the patient was diabetic and had a deranged blood sugar level of $231 \mathrm{mg} / \mathrm{dl}$ with chronic kidney disease and serum creatine 2.2 $\mathrm{mg} / \mathrm{dl}$. In Case 2 in the $1^{\text {st }}$ episode patient had deranged Inflammatory markers CRP and DDimer are positive however in the 2nd episode both the markers were negative. In Case 3 in the 1 st episode Inflammatory markers i.e. CRP and D-Dimer are negative however in the $2^{\text {nd }}$ episode only D-Dimer was positive. In cases 4 and 5 no laboratory investigations were done in the 1 st episode however in the $2^{\text {nd }}$ episode inflammatory markers were negative.

\section{DISCUSSION}

SARS-CoV-2 was first expected to induce a monophasic disease with at least transient immunity [1,2]. Nevertheless, rare cases of suspected Covid-19 "recurrence" or "reactivation" have been reported [3]. Immunity to SARS-CoV-2 involves both cell-mediated and humoral responses, but its protective role from re-infection along with definitive viral clearance is uncertain [4]. This case series of five patients having experienced two separate Covid-19 episodes, associated with viral detection, raises two hypotheses underlying these recurrences: viral reinfection or viral reactivation from the given data.

Covid-19 recurrences should be differentiated from the persistence of traces of viral RNA that can be detected in respiratory samples up to 6 weeks after onset of symptoms in clinicallycured patients [5). An inflammatory rebound triggered by an inappropriate immune response could constitute an alternative explanation to the recurrence of clinical symptoms [6].

In the cases (Cases 2, 3, and 5) with symptoms at both episodes, a re-infection due to the prolonged exposition can be supposed [7]. Also, in the patient (Case 4) being symptomatic in 2nd episode, a re-infection can be a consideration due to prolonged exposure [7].

Immunosuppressive factors such as drugs or pathological conditions could contribute to impair 
viral clearance and favor SARS-CoV-2 reactivation as in the case of the man (Case 1) had Immunosuppressive factors such Diabetes mellitus type II and Chronic kidney disease could contribute to impair viral clearance and favor SARS-CoV-2 reactivation [8]. During the 1 st episode, the patient was asymptomatic and was home quarantine during the 2nd episode required ICU hospitalization, with the patient still being under treatment. He was on Remdesivir, Methylprednisolone, low molecular weight heparin, and oxygen therapy during the $2^{\text {nd }}$ episode.

In conclusion, the above case series of the patients they are re-infected, with potential longterm effects of the immunosuppressive state that can hamper the immune response, constitute a substantial point of vigilance for the management of the pandemic at the individual and collective levels. Studies including genomic comparisons of viral strains, determination of RNA infectivity by viral culture, as well as assessment of innate and adaptive immunity and monitoring inflammatory targets, would be of great value for further understanding the underlying pathophysiology of these Covid-19 recurrences.

\section{ACKNOWLEDGMENTS}

Declaration of Conflicting Interests: The authors declare that they have no conflict of interest.

\section{Funding: Not applicable}

\section{REFERENCES}

1. Kiyuka PK, Agoti CN, Munywoki PK, et al. Human coronavirus NL63 molecular epidemiology and evolutionary patterns in rural coastal Kenya. J Infect Dis. 2018; 217(11):1728-1739.

2. Tang $F$, Quan $Y$, Xin ZT, et al. Lack of peripheral memory $\mathrm{B}$ cell responses in recovered patients with severe acute respiratory syndrome: a six-year followup study. J Immunol. 2011; 186(12):7264-7268.

3. Ye G, Pan Z, Pan Y, et al. Clinical characteristics of severe acute respiratory syndrome coronavirus 2 reactivation. J Infect 2020 80(5):e14-e17.

4. Grifoni A, Weiskopf D, Ramirez SI, et al. Targets of $\mathrm{T}$ cell responses to SARS-CoV-2 coronavirus in humans with COVID-19 disease and unexposed individuals. Cell 2020;181(7):1489-1501.e15.

5. Xiao AT, Tong YX, Zhang S. Profile of RT-PCR for SARS-CoV-2: a preliminary study from 56 COVID-19 patients. Clin Infect Dis 2020; 19;71(16):2249-2251.
6. Gousseff $M$, Penot $P$, Gallay $L$, et al. Clinical recurrences of COVID-19 symptoms after recovery: viral relapse, reinfection or inflammatory rebound? J Infect 2020; 81(5):816-846.

7. Zhao J, Yuan $Q$, Wang $H$, et al. Antibody responses to SARS-CoV-2 in patients of novel coronavirus disease 2019. Clin Infect Dis. 2020; 71(16):2027-2034.

8. Ling $Y, X u$ SB, Lin $Y X$, et al. Persistence and clearance of viral RNA in 2019 novel coronavirus disease rehabilitation patients. Chin Med J (Engl) 2020; 133(9):1039-1043. 